\title{
The impact of social media in business growth and performance: A scientometrics analysis
}

\author{
A. Pourkhania ${ }^{\mathrm{a}}$, Kh. Abdipour ${ }^{\mathrm{a}}$, B. Baher ${ }^{\mathrm{a}}$ and M. Moslehpour ${ }^{\mathrm{a}, \mathrm{b}^{*}}$
}

\begin{tabular}{l}
${ }^{a}$ Department of Business Administratio \\
${ }^{b}$ Department of Business Administratio \\
\hline C H R O N I C L E \\
\hline Article history: \\
Received: October 28, 2018 \\
Received in revised format: Janu- \\
ary 29, 2019 \\
Accepted: February 5, 2019 \\
Available online: \\
February 6, 2019 \\
\hline Keywords: \\
Social media \\
Business \\
Social network \\
Marketing
\end{tabular}

\section{Introduction}

The concept of Social Media (SM) has been on top of the agenda for many business executives. Decision makers, as well as consultants, try to identify ways in which firms can make profitable use of applications such as Wikipedia, YouTube, Facebook, Twitter, etc. (Kaplan \& Haenlein 2010). Traditionally, consumers used the Internet to simply expend content: they read it, they watched it, and they used it to buy products and services. This represents the social media phenomenon, which can now significantly impact a firm's reputation, sales, and even survival. Yet, many executives eschew or ignore this form of media because they do not understand what it is, the various forms it can take, and how to engage with it and hoe to learn (Kietzmann et al., 2011). Today social media platforms such as Twitter and Facebook enable the creation of virtual customer environments (VCEs) where online communities of interest form around specific firms, brands, or products. (Culnan et al., 2010).

\footnotetext{
* Corresponding author.

E-mail address: writetodrm@gmail.com (M. Moslehpour)

(C) 2019 by the authors; licensee Growing Science, Canada. doi: $10.5267 /$ j.ijdns.2019.2.003 
The emergence of Internet-based SM has made it possible for anyone to communicate with literally thousands of people about particular products and the companies that provide them. Thus, the impact of consumer-to-consumer communications has been widely magnified in the marketplace. SM is a hybrid element of the promotion mix because in a traditional sense it helps firms talk to their customers, while in a nontraditional sense it only assists customers to talk directly to one another. It is often outside managers' direct control to monitor the content and the media-based conversations happening between consumers. This is in contrast to the conventional marketing communications paradigm whereby a high degree of control exists. Thus, managers ought to learn to form consumer discussions in a way that is complied with the organization's mission and performance objectives. Methods by which this can be achieved are delineated herein (Mangold \& Faulds, 2009). According to Berthon et al. (2012), to help managers learn more about this new dispensation five axioms are needed to follow: (1) SMs always follow a technology, culture, and government of a particular country or context; (2) local events seldom are local; (3) global events most likely to be (re)interpreted locally; (4) creative consumers' actions and creations depend on technology, culture, and government; and (5) technology is historically dependent. Social media explores the cultural landscape of open source branding, and identifies marketing strategies directed at the hunt for consumer engagement on the People's Web. These strategies exhibit a paradox to reach coveted resonance and the brand has to relinquish control (Fournier \& Avery, 2011). All this has helped companies make significant changes on their business strategies. Benefits from the implementation of SM sites increase in awareness and inquiries and enhanced relationships with customers. An increase in the number of new customers has enhanced the ability to reach customers on a global scale and co-promotion of local businesses which enhance the image of small businesses in the region (Jones et al., 2015). With the introduction of Web 2.0, consumers have been more interested in expressing and sharing their ideas on web regarding day-to-day activities and global issues as well. Evolution of SM has also attributed to such activities by giving us a transparent platform to share their views around the world. These electronic Word of Mouth (eWOM) statements expressed on the web are much prevalent in business and service industry to help consumers share their point of view (Ravi \& Ravi, 2015). Thus, a large amount of usergenerated content becomes available on SM sites. To increase competitive advantage and effectively reach the competitive environment of businesses, most firms require to carefully monitor and analyze both the customer-generated content on their own social media sites and the textual information on their competitors' SM sites (He et al., 2013). The objective is to find the necessary information from this complex data and implement it for trend analysis and prediction. Visual analytics method applied by the toolkit can be used in other domains involving SM data, such as sales prediction and advertisement planning (Lu et al., 2014). Customer feedback which appear in social media, however, are basically unstructured, therefore, a large data set is required for meaningful analysis. Although determining consumers' value structures and behaviors is useful for developing different markets, the unstructured and volumeheavy nature of consumer data bans effective and economical extraction of such information (Jang et al., 2013). The social networking application is not merely associated with customer relationships then relying on the industry, the objectives, etc., and managers have to choose their own techniques. Finally, some

have attempted to standardize the implementation of SM networks for businesses by building some necessary tools (Colomo-Palacios et al., 2014).

\section{About Bibliometrix R package}

Bibliometrics is the application of quantitative analysis and statistics to publications such as journal articles and their accompanying citation counts. Quantitative evaluation of publication and citation data is now used in almost all science fields to evaluate growth, maturity, leading authors, conceptual and intellectual maps and the trend of a scientific community. Bibliometrics is also used in research performance evaluation (Aria \& Cuccurullo, 2017).

\section{Annual scientific production}

With the fast development of social networks, scientific articles on this topic have also grown dramatically. Here we will review 2682 scientific articles to examine the dimensions of this topic. 


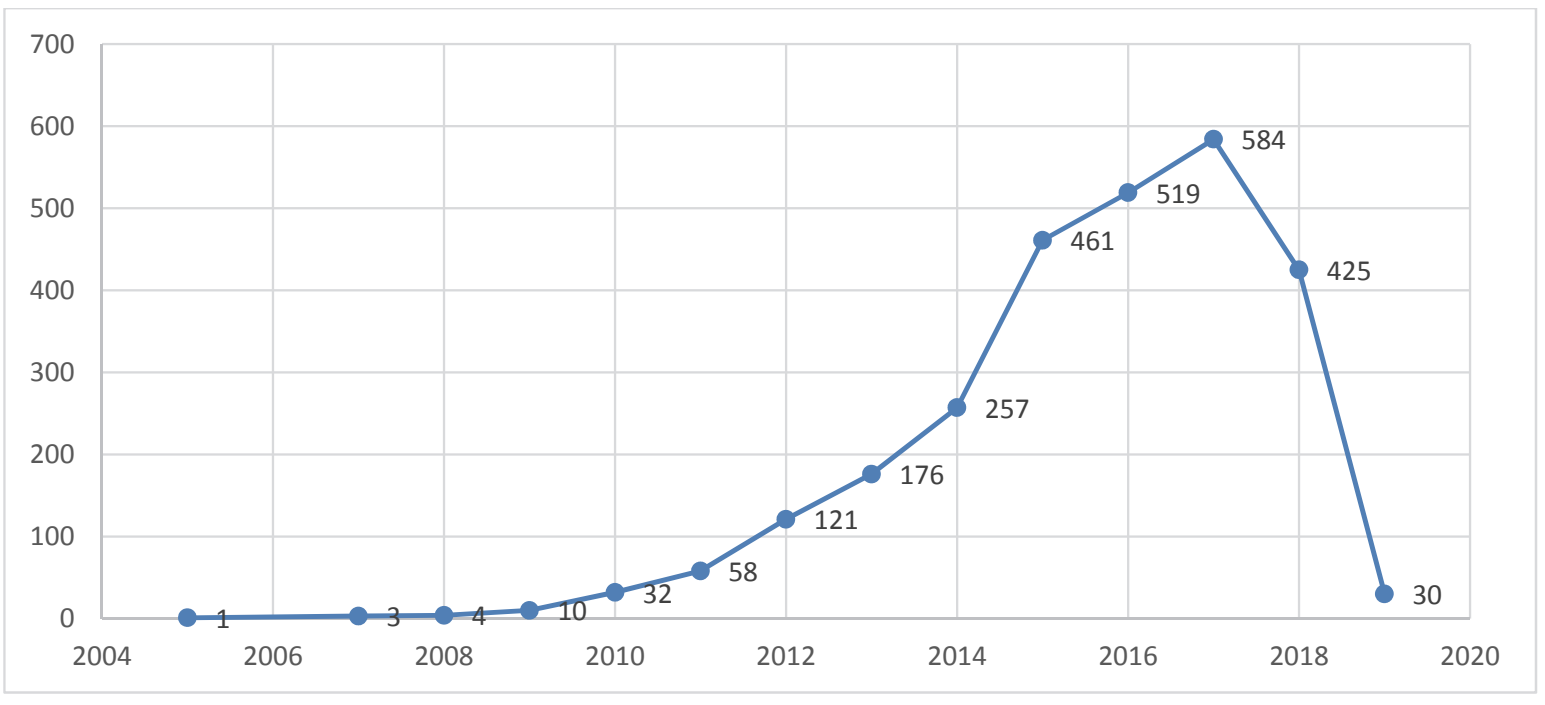

Fig. 1. The Web of Science publications on the analysis of social media and business from 2005 to 2019

Fig. 1 shows the growth trend of scientific content in the field of social media and business. It shows that in 2014 we witnessed a mutation.

\section{Source Dynamics}

Fig. 2 shows that the Business Horizons Magazine has a significant contribution to the publication of this topic and, compared to other journals, is highly specialized in the subject of business.

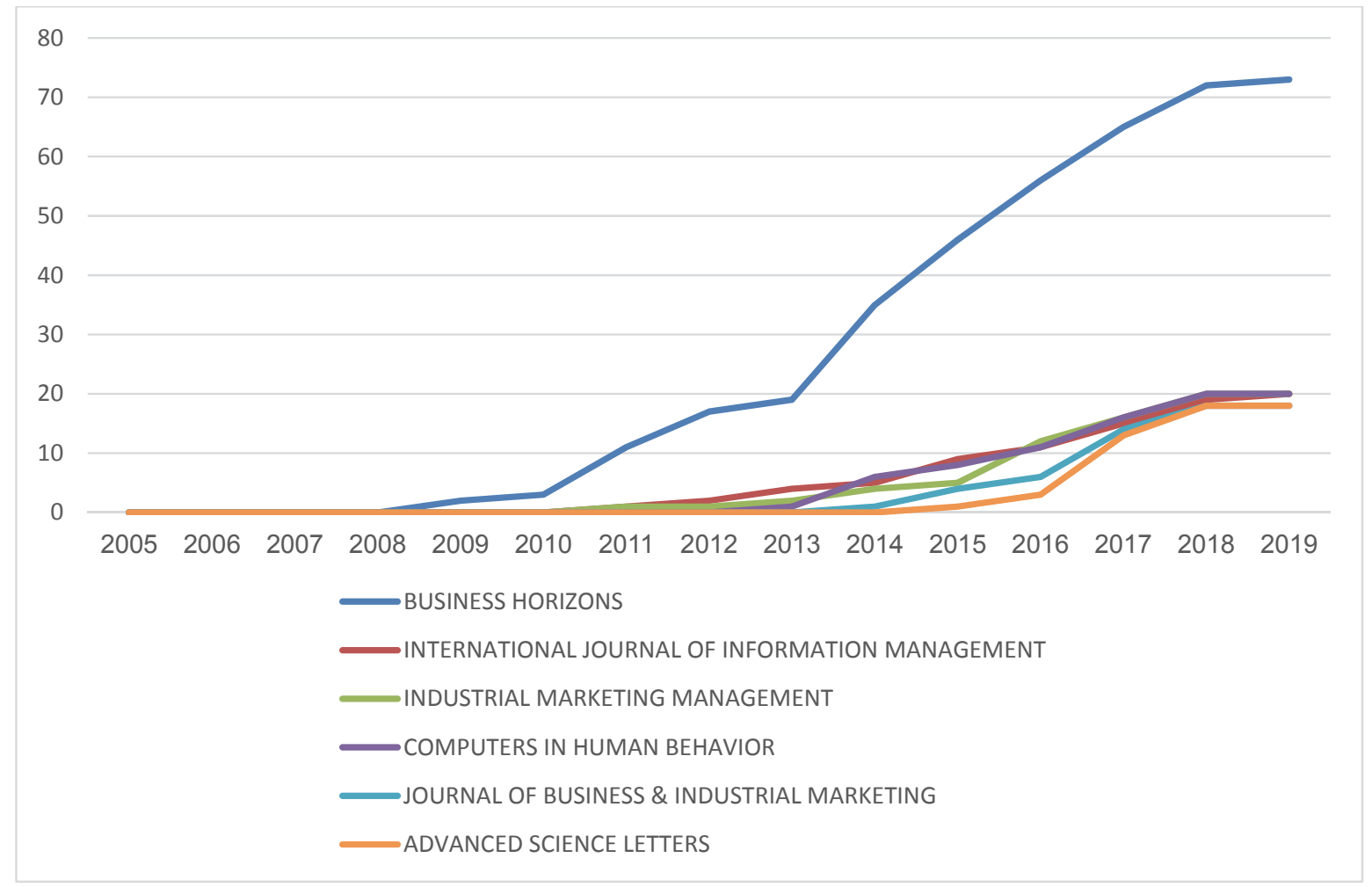

Fig. 2. Trend of content related to the subject at different scientific journals 


\section{Country Scientific Production}

One of the other important areas of research is the study of the scientific production of different countries. Studies show that researchers from the United States (1269 articles), UK (364 article), China (309 articles) and the Australia (265) have played a major role in scientific production of social media and business.

Table 1

Country scientific production in studies with social media in business

\begin{tabular}{llll}
\hline \multicolumn{1}{c}{ region } & Freq & \multicolumn{1}{c}{ region } & Frea \\
\hline USA & 1269 & GREECE & 47 \\
UK & 364 & BRAZIL & 45 \\
CHINA & 309 & THAILAND & 42 \\
AUSTRALIA & 265 & DENMARK & 39 \\
INDIA & 254 & NORWAY & 39 \\
SPAIN & 179 & POLAND & 38 \\
GERMANY & 139 & SOUTH AFRICA & 37 \\
CANADA & 132 & CZECH REPUBLIC & 34 \\
MALAYSIA & 125 & SWITZERLAND & 34 \\
ITALY & 120 & SAUDI ARABIA & 33 \\
PORTUGAL & 98 & PAKISTAN & 32 \\
TAIWAN & 98 & SINGAPORE & 31 \\
ROMANIA & 96 & JAPAN & 27 \\
FINLAND & 95 & RUSSIA & 26 \\
SOUTH KOREA & 95 & AUSTRIA & 25 \\
INDONESIA & 93 & SLOVAKIA & 25 \\
FRANCE & 82 & BELGIUM & 24 \\
TURKEY & 75 & IRELAND & 24 \\
NETHERLANDS & 59 & IRAN & 23 \\
SWEDEN & 59 & NEW ZEALAND & 22 \\
\hline
\end{tabular}

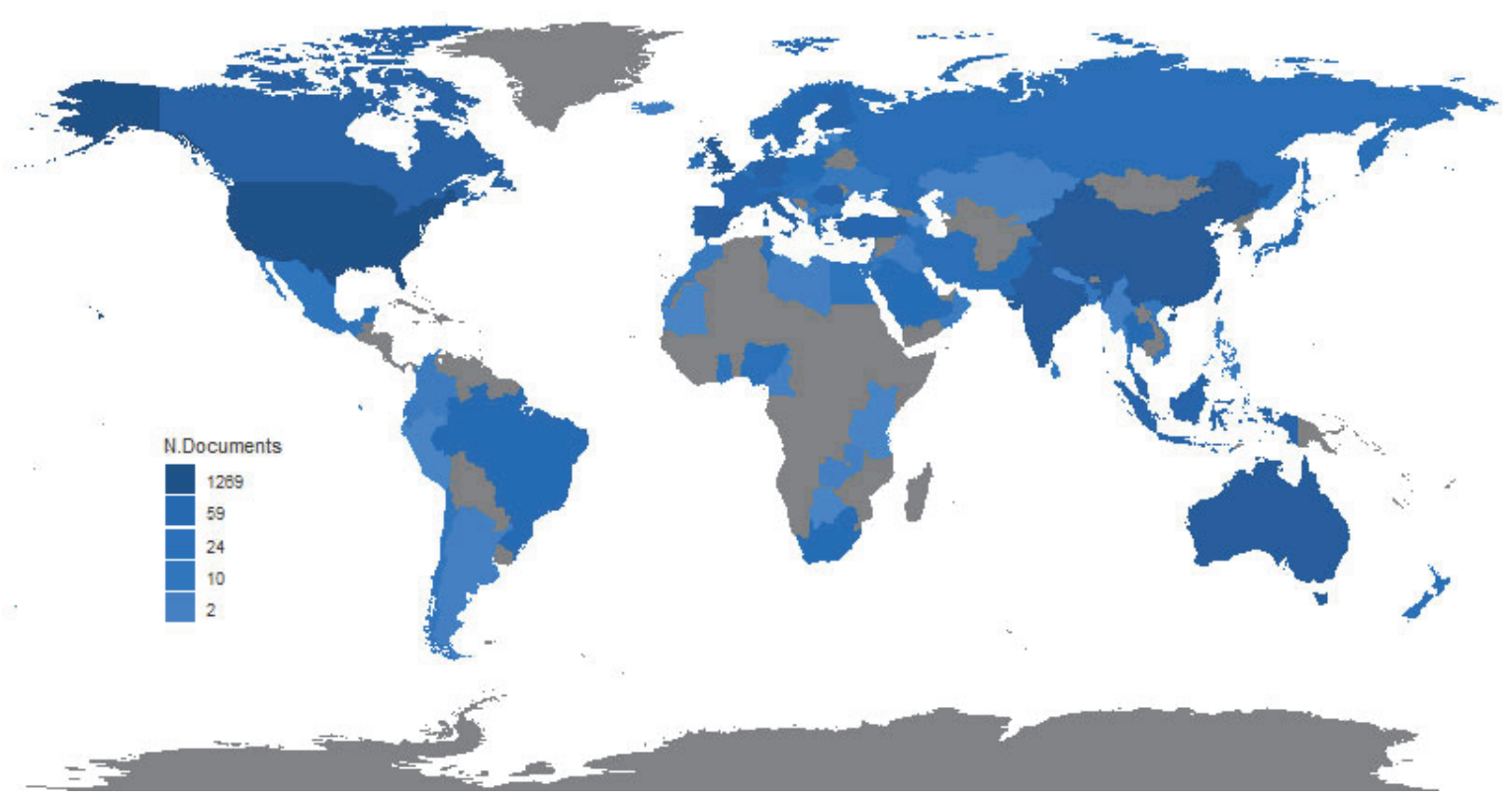

Fig. 3. Country scientific production on map

\section{Corresponding Author's Country}

Corresponding Author's Country shows how the authors have been collaborating in one country, or different countries in subject. 
SCP: Single Country Publication

MCP: Multi Country Publication

Fig. 4 illustrates the collaboration of the authors from different countries on this topic.

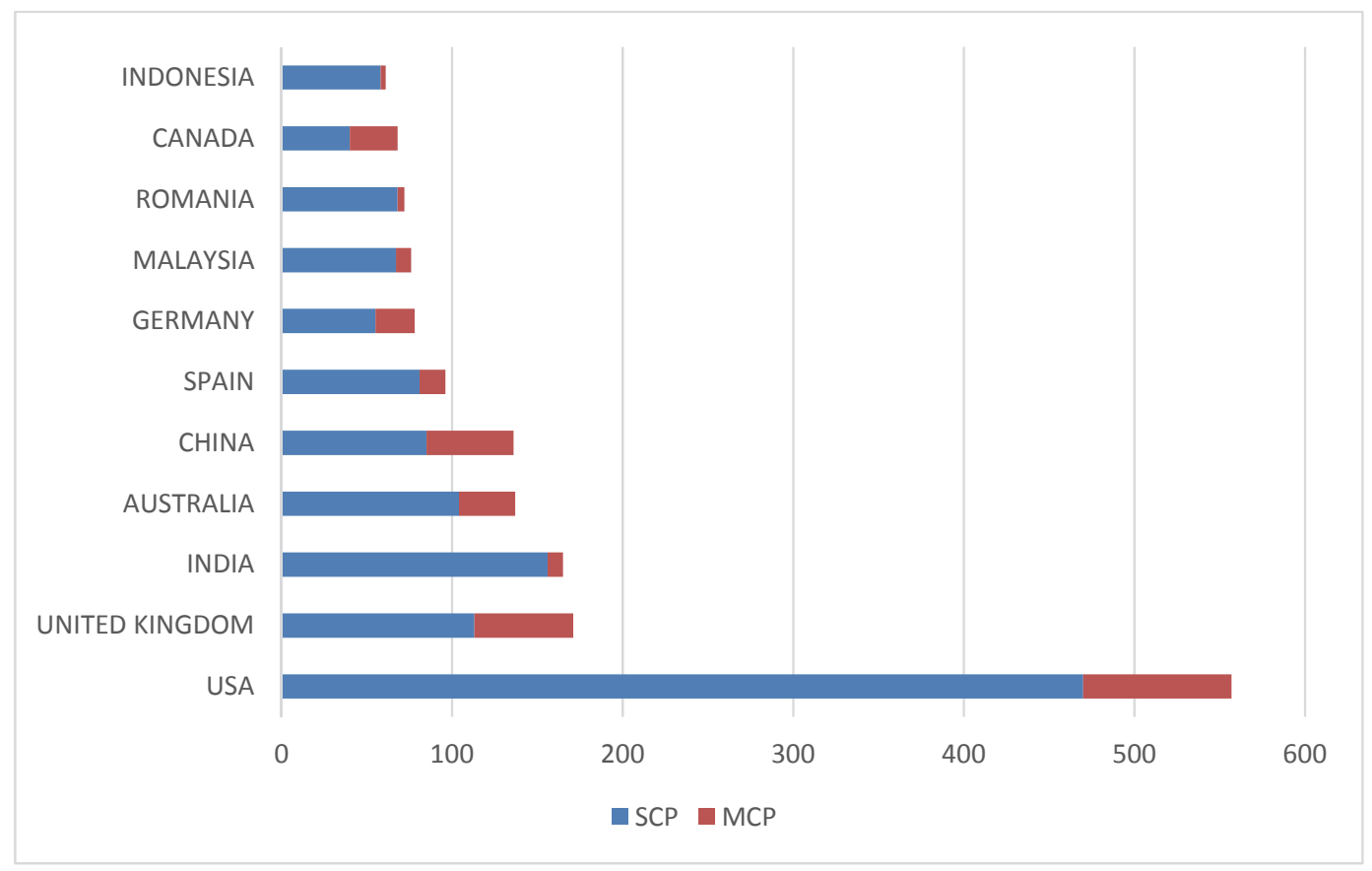

Fig. 4. Corresponding Author's Country

\section{Most Cited Countries}

The study of the countries influencing the production of science shows that the United States is the leader in this field, followed by France, Canada and the United Kingdom. The results show that leading countries cover more than $70 \%$ of the total number of references.

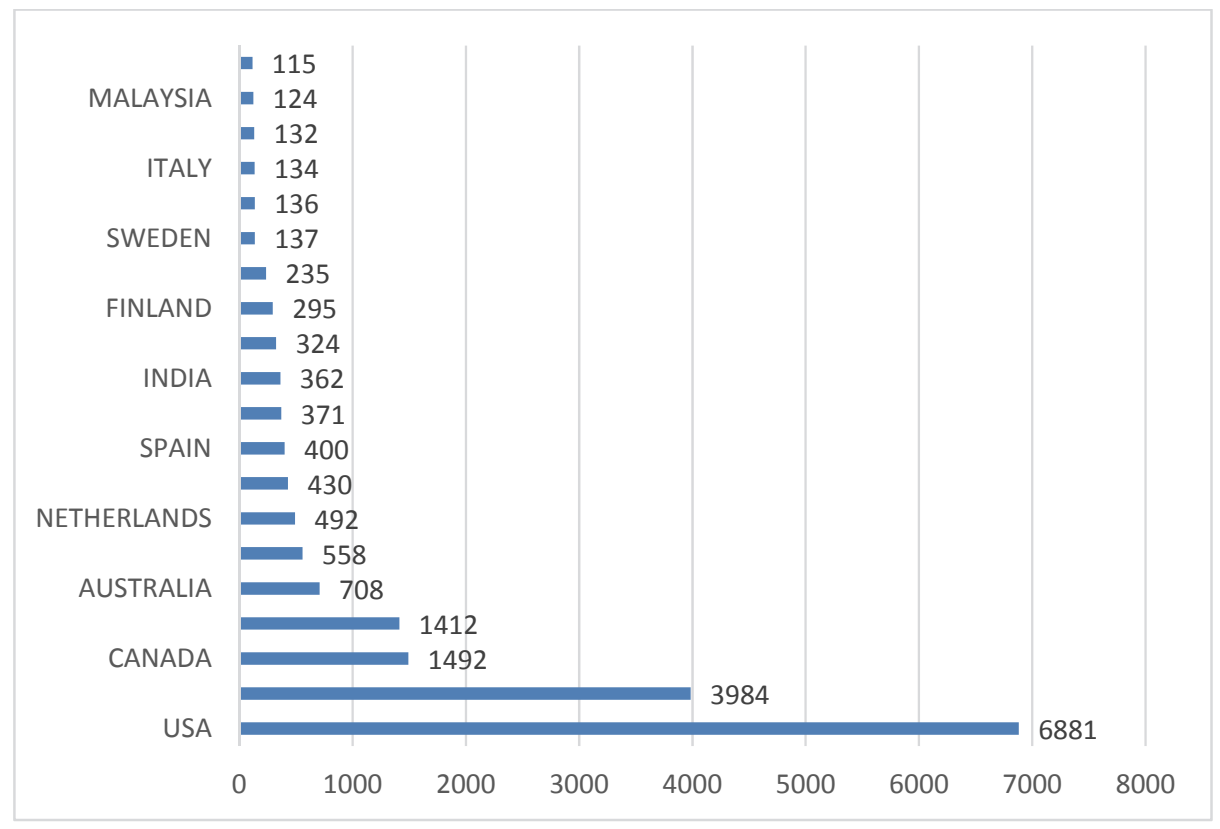

Fig. 5. Most Cited Countries 


\section{The most common keywords and Temporal Analysis}

Table 2 demonstrates some of the most popular keywords used in studies associated with Social media and business. As we can observe from the results of Table 2, "social media", "impact" and "word-ofmouth" are three keywords known in the literature. Fig. 6 shows the most important words used over times.

Table 2

The most popular keywords used in studies with social media in business.

\begin{tabular}{|c|c|c|c|}
\hline Words & Occurrences & Words & Occurrences \\
\hline social media & 261 & antecedents & 27 \\
\hline impact & 171 & business intelligence & 26 \\
\hline word-of-mouth & 156 & competitive advantage & 26 \\
\hline performance & 140 & customer satisfaction & 26 \\
\hline model & 123 & strategies & 26 \\
\hline management & 122 & acceptance & 24 \\
\hline information & 112 & challenges & 24 \\
\hline internet & 107 & firm & 24 \\
\hline perspective & 104 & hospitality & 24 \\
\hline networks & 93 & smes & 24 \\
\hline innovation & 92 & user acceptance & 24 \\
\hline behavior & 90 & power & 23 \\
\hline business & 87 & user-generated content & 22 \\
\hline information-technology & 87 & brand community & 21 \\
\hline media & 84 & consumer & 21 \\
\hline framework & 79 & design & 21 \\
\hline Facebook & 76 & e-commerce & 21 \\
\hline communication & 75 & firms & 21 \\
\hline technology & 75 & technologies & 21 \\
\hline trust & 72 & work & 21 \\
\hline adoption & 67 & capabilities & 20 \\
\hline twitter & 64 & co-creation & 20 \\
\hline online & 59 & classification & 19 \\
\hline systems & 53 & commitment & 19 \\
\hline communities & 52 & future & 19 \\
\hline web & 51 & knowledge management & 19 \\
\hline knowledge & 49 & models & 19 \\
\hline organizations & 49 & community & 18 \\
\hline engagement & 46 & context & 18 \\
\hline quality & 45 & dynamic capabilities & 18 \\
\hline satisfaction & 43 & e-business & 18 \\
\hline consumers & 39 & environments & 18 \\
\hline sales & 38 & governance & 18 \\
\hline big data & 35 & intelligence & 18 \\
\hline determinants & 35 & intention & 18 \\
\hline industry & 34 & networking sites & 18 \\
\hline strategy & 34 & relationship quality & 18 \\
\hline loyalty & 33 & service quality & 18 \\
\hline participation & 33 & services & 18 \\
\hline sites & 32 & support & 18 \\
\hline brand & 31 & companies & 17 \\
\hline information-systems & 31 & consumption & 17 \\
\hline online communities & 31 & education & 17 \\
\hline reviews & 30 & experience & 17 \\
\hline usage & 30 & orientation & 17 \\
\hline firm performance & 29 & customer relationship management & 16 \\
\hline web 2.0 & 29 & higher-education & 16 \\
\hline analytics & 28 & identification & 16 \\
\hline sentiment analysis & 28 & identity & 16 \\
\hline tourism & 28 & network & 16 \\
\hline
\end{tabular}


As shown in Fig. 6, "social media", "business", "data", "marketing", "management", "analysis", "communication", "content" and "customer" are the research hotspots with a high frequency of the keywords used in different project.

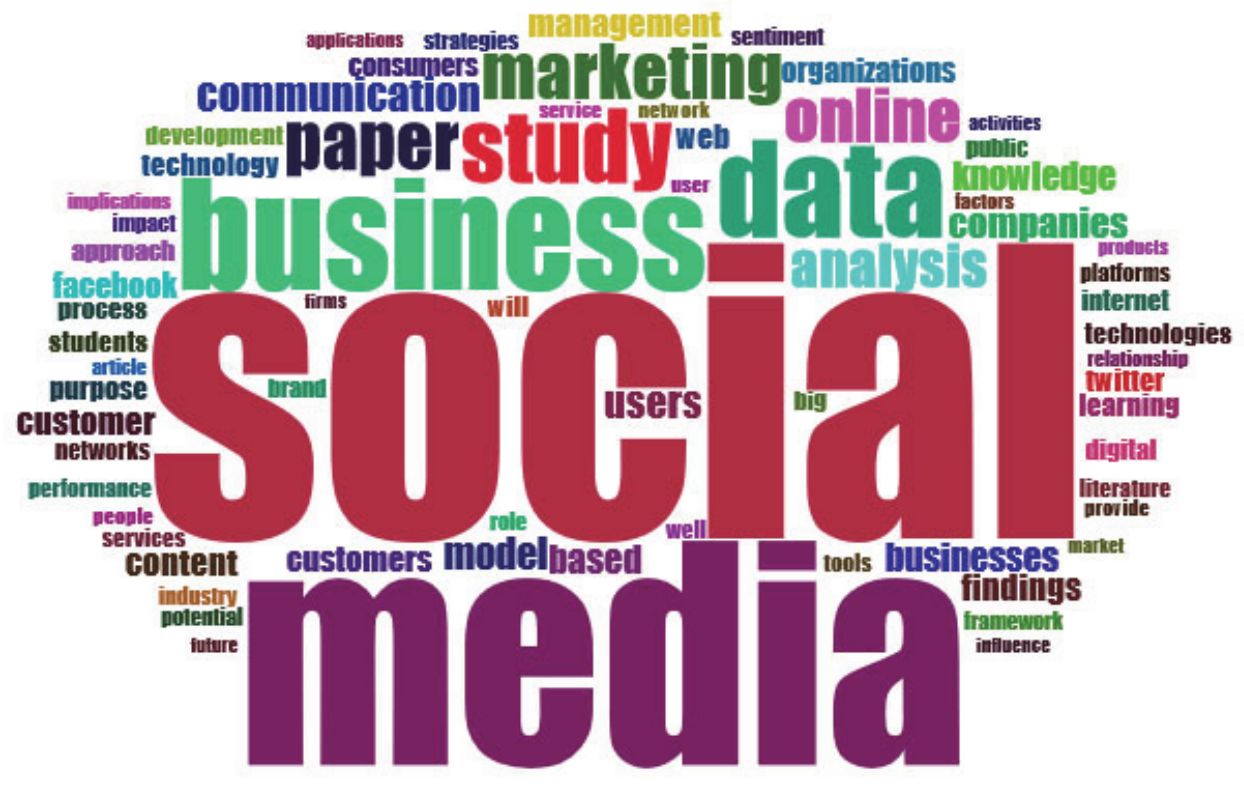

Fig. 6. The frequency of the keywords used in different paper

\section{Conceptual structure, Co-occurrence network}

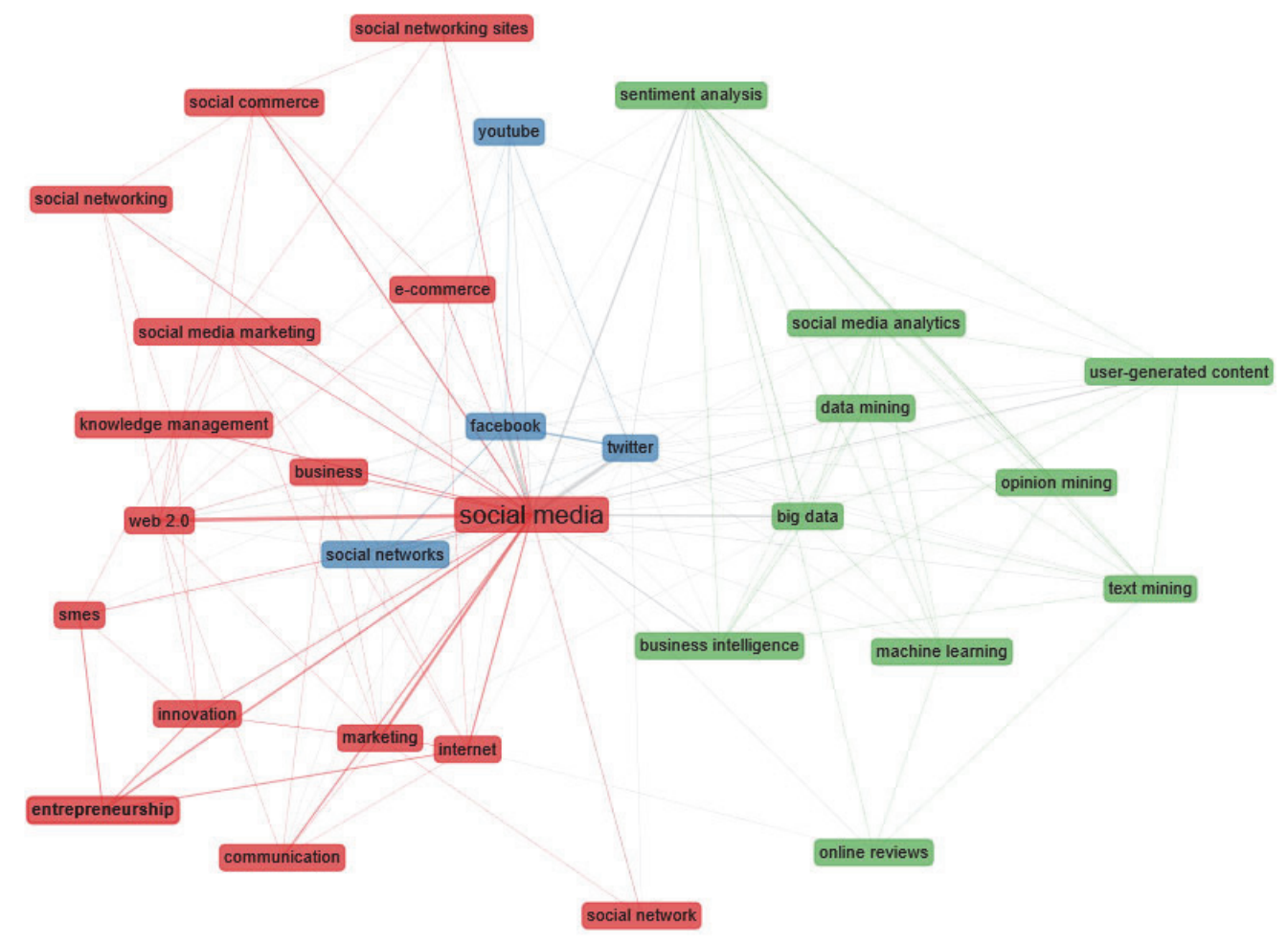

Fig. 7. Co-occurrence network (2005-2019) 
A keywords co-occurrence network $(\mathrm{KCN})$ focuses on understanding the knowledge components and knowledge structure of a scientific/technical field by examining the links between the keywords in the literature. Fig. 7 focuses on the analysis methods based on KCNs, which have been used in theoretical and empirical studies to explore research topics and their relationships in selecting scientific fields. If keywords are grouped into the same cluster, they are more likely to reflect identical topics. Each cluster has different number of subject keyword. As can be seen from the Fig.7, the social networking platform, data and its use, and social media applications are the main clusters of this research.

\section{Conceptual structure map, Correspondence analysis}

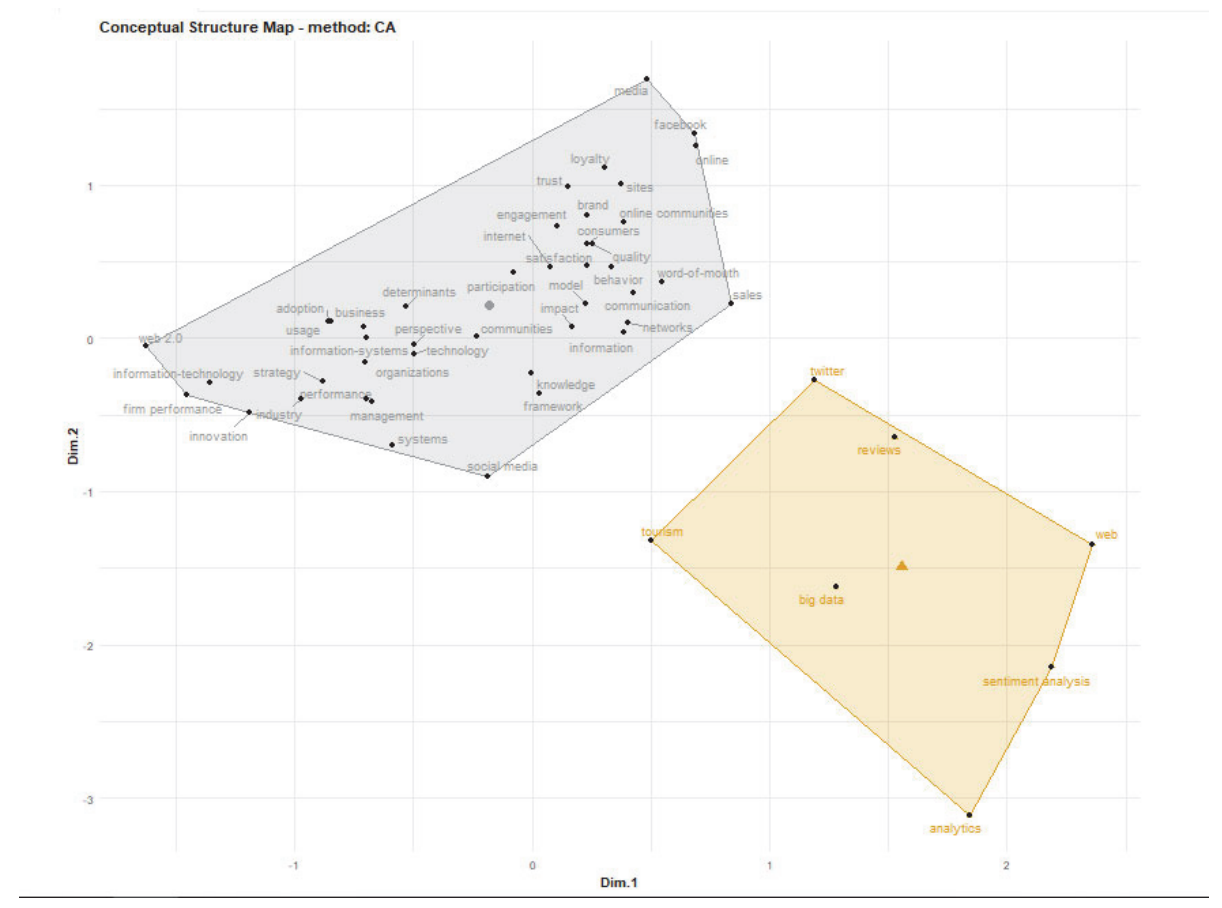

Fig. 8. Co-occurrence network (2005-2019)

Co-word analysis aims at representing the conceptual structure of a framework using co-occurrence of words. The words can be replaced by authors' keywords, keywords plus, and terms extracted from titles or abstracts. The conceptual structure function produces three kinds of mapping as listed: conceptual structure map, factorial map of the documents with the highest contributes and factorial map of the most cited documents. Conceptual structure map is presented in Fig. 8. Left Cluster has the most keywords, which means the attention of the researchers to the subject matter of the study.

\section{Thematic map}

Co-word analysis draws clusters of the keywords and they are considered as themes. In the strategic diagram presented in Fig. 9, the vertical axis measures the density - i.e., the strength of the internal links within a cluster represented by a theme -, and the horizontal vertical axis the centrality - i.e. the strength of the links between the theme and other themes in the map. Thematic map is a very intuitive plot and we can analyze themes according to the quadrant in which they are placed:

(Q1) upper-right quadrant: motor-themes

(Q2) lower-right quadrant: basic themes

(Q3) lower-left quadrant: emerging or disappearing themes;

(Q4) upper-left quadrant: very specialized/ niche themes. 


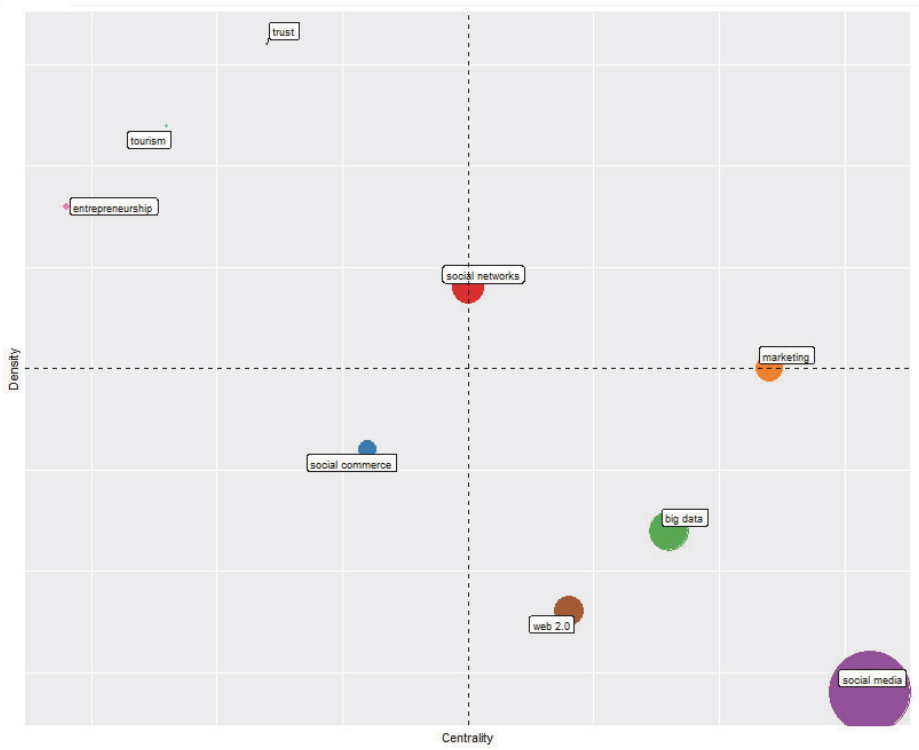

Fig.9. Thematic Map

Hence, the themes with the highest internal coherence and closest relationship to other themes appear in the second quadrant (the lower right part of the graph). In the third and fourth, the following topics can be found: social commerce, social networking, entrepreneurship and tourism. Themes in this quadrant are important for a research field but are not developed. This quadrant group's transversal and general, basic themes.

\section{Social structure, Contributions of countries}

As shown in Fig. 10, the United States is the leader in science production in this topic.

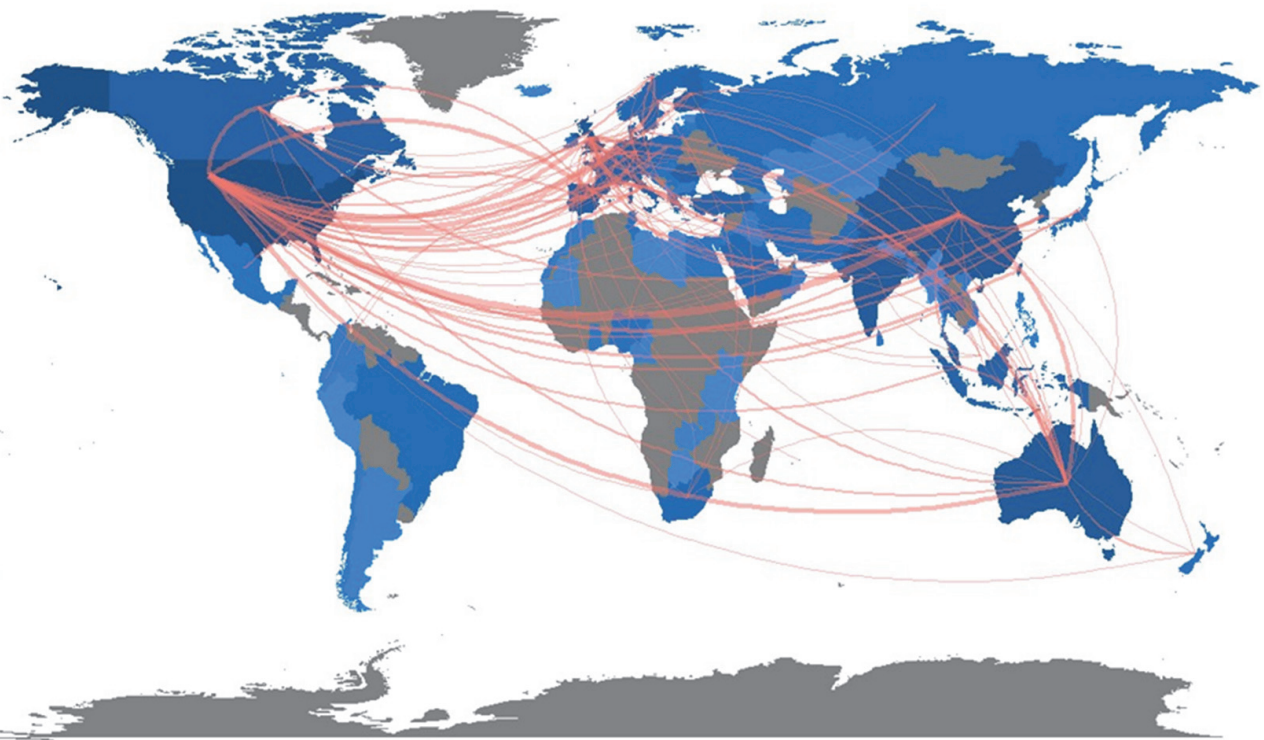

Fig. 10. Country collaboration 
Table 3

Country collaboration in studies about social media in business

\begin{tabular}{|c|c|c|}
\hline From & To & Frequency \\
\hline USA & CHINA & 47 \\
\hline CANADA & USA & 22 \\
\hline USA & UNITED KINGDOM & 18 \\
\hline UNITED KINGDOM & ITALY & 15 \\
\hline USA & AUSTRALIA & 15 \\
\hline AUSTRALIA & CHINA & 14 \\
\hline FRANCE & USA & 14 \\
\hline FRANCE & UNITED KINGDOM & 12 \\
\hline UNITED KINGDOM & CHINA & 12 \\
\hline USA & GERMANY & 12 \\
\hline USA & TAIWAN & 12 \\
\hline USA & FINLAND & 11 \\
\hline AUSTRALIA & UNITED KINGDOM & 10 \\
\hline USA & NETHERLANDS & 10 \\
\hline USA & ITALY & 8 \\
\hline USA & KOREA & 8 \\
\hline CANADA & AUSTRALIA & 7 \\
\hline CANADA & CHINA & 7 \\
\hline CANADA & UNITED KINGDOM & 7 \\
\hline NETHERLANDS & UNITED KINGDOM & 7 \\
\hline UNITED KINGDOM & GERMANY & 7 \\
\hline USA & INDIA & 7 \\
\hline AUSTRALIA & GERMANY & 6 \\
\hline DENMARK & NORWAY & 6 \\
\hline FRANCE & AUSTRALIA & 6 \\
\hline CHINA & KOREA & 5 \\
\hline FRANCE & GERMANY & 5 \\
\hline ITALY & SPAIN & 5 \\
\hline SPAIN & PORTUGAL & 5 \\
\hline UNITED KINGDOM & SPAIN & 5 \\
\hline USA & BELGIUM & 5 \\
\hline USA & NORWAY & 5 \\
\hline USA & SINGAPORE & 5 \\
\hline USA & SPAIN & 5 \\
\hline USA & SWITZERLAND & 5 \\
\hline AUSTRALIA & DENMARK & 4 \\
\hline AUSTRALIA & NEW ZEALAND & 4 \\
\hline AUSTRALIA & SINGAPORE & 4 \\
\hline
\end{tabular}

\section{Highly cited papers}

Although articles' citation is considered as an indicator of the impact of papers, the impact of the article cannot be evaluated solely by considering the first influential articles. More people have not yet seen newer articles that are truly influential and, therefore, they have not shown their influence. Table 4 shows the summary of the most cited articles. As we can observe from the results of Table 3 , the study by Kaplan and Haenlein (2010) has received the highest citations. This paper analyzed the challenges and opportunities of Social Media. They provided a classification of Social Media, which group applications currently subsumed under the generalized term into more specific categories by different characteristics such as collaborative projects, blogs, content communities, social networking sites, virtual game worlds, and virtual social worlds. Finally, they presented 10 pieces of advice for companies, which decide to utilize Social Media. The second highly cited work belongs to Kietzmann et al. (2011), which explains that the SM now significantly influences on a firm's reputation, sales, and even survival. Yet, many business partners disregard this form of media since they have not realized the value of this market, the different forms it can take, and how to engage with it and learn. They provided a framework which 
defines SM by using seven functional building blocks including identity, conversations, sharing, presence, relationships, reputation, and groups. They also suggested on how firms should develop different strategies for monitoring, understanding, and responding to various social media activities. The third highly cited work is associated with Mangold and Faulds (2009) where the focus was on communication in social networks. In addition, they emphasized that social networks for companies have two parts, the first is the activities that are accomplished by the company, and the second is the ones that users share about the firms. Therefore, managers have to learn to direct consumer interests to the mission and objectives of the organizations. Then they introduced methods for this purpose.

Table 4

The summary of the most cited articles

\begin{tabular}{|c|c|c|}
\hline Paper & Total Citations & TC per Year \\
\hline KAPLAN AM, 2010, BUS HORIZONS & 3332 & 370.2 \\
\hline KIETZMANN JH, 2011, BUS HORIZONS & 977 & 122.1 \\
\hline MANGOLD WG, 2009, BUS HORIZONS & 804 & 80.4 \\
\hline HANNA R, 2011, BUS HORIZONS & 379 & 47.4 \\
\hline SASHI CM, 2012, MANAGE DECIS & 235 & 33.6 \\
\hline BERTHON PR, 2012, BUS HORIZONS & 228 & 32.6 \\
\hline VERHOEF PC, 2015, J RETAILING & 214 & 53.5 \\
\hline CULNAN MJ, 2010, MIS Q EXEC & 211 & 23.4 \\
\hline FOURNIER S, 2011, BUS HORIZONS & 204 & 25.5 \\
\hline HE W, 2013, INT J INFORM MANAGE & 199 & 33.2 \\
\hline MICHAELIDOU N, 2011, IND MARKET MANAG & 192 & 24.0 \\
\hline ARAL S, 2013, INFORM SYST RES & 181 & 30.2 \\
\hline MUNAR AM, 2014, TOURISM MANAGE & 171 & 34.2 \\
\hline RAVI K, 2015, KNOWL-BASED SYST & 165 & 41.3 \\
\hline LIANG TP, 2011, INT J ELECTRON COMM & 164 & 20.5 \\
\hline KIM W, 2010, INFORM SYST & 155 & 17.2 \\
\hline KAVANAUGH AL, 2012, GOV INFORM Q & 146 & 20.9 \\
\hline MALTHOUSE EC, 2013, J INTERACT MARK & 143 & 23.8 \\
\hline LUO XM, 2013, INFORM SYST RES & 129 & 21.5 \\
\hline WEINBERG BD, 2011, BUS HORIZONS & 126 & 15.8 \\
\hline LI LN, 2013, CARTOGR GEOGR INF SC & 119 & 19.8 \\
\hline TRAINOR KJ, 2014, J BUS RES & 116 & 23.2 \\
\hline DU SL, 2012, J BUS ETHICS & 115 & 16.4 \\
\hline KAPLAN AM, 2011, BUS HORIZONS & 115 & 14.4 \\
\hline SOTIRIADIS MD, 2013, ELECTRON COMMER RES & 113 & 18.8 \\
\hline RISHIKA R, 2013, INFORM SYST RES & 108 & 18.0 \\
\hline KAPLAN AM, 2012, BUS HORIZONS & 106 & 15.1 \\
\hline KAPLAN AM, 2011, BUS HORIZONS-a & 103 & 12.9 \\
\hline DAVENPORT TH, 2012, MIT SLOAN MANAGE REV & 98 & 14.0 \\
\hline RAPP A, 2013, J ACAD MARKET SCI & 96 & 16.0 \\
\hline XIANG Z, 2015, J RETAIL CONSUM SERV & 94 & 23.5 \\
\hline YU Y, 2013, DECIS SUPPORT SYST & 93 & 15.5 \\
\hline KWOK L, 2013, CORNELL HOSP Q & 92 & 15.3 \\
\hline SCOTT SV, 2012, ACCOUNT ORG SOC & 91 & 13.0 \\
\hline KAPLAN AM, 2009, BUS HORIZONS & 89 & 8.9 \\
\hline XIE KL, 2014, INT J HOSP MANAG & 87 & 17.4 \\
\hline ZHOU LN, 2013, ELECTRON COMMER R A & 87 & 14.5 \\
\hline LARISCY RW, 2009, PUBLIC RELAT REV & 87 & 8.7 \\
\hline PANTELIDIS IS, 2010, CORNELL HOSP Q & 84 & 9.3 \\
\hline SAXTON GD, 2013, INFORM SYST MANAGE & 83 & 13.8 \\
\hline FERRARA E, 2014, KNOWL-BASED SYST & 81 & 16.2 \\
\hline CHAE B, 2015, INT J PROD ECON & 77 & 19.3 \\
\hline LIN KY, 2011, CYBERPSYCH BEH SOC N & 77 & 9.6 \\
\hline CHENG MM, 2016, INT J HOSP MANAG & 76 & 25.3 \\
\hline HAJLI MN, 2014, TECHNOL FORECAST SOC & 75 & 15.0 \\
\hline PIOTROWICZ W, 2014, INT J ELECTRON COMM & 73 & 14.6 \\
\hline ABRAHAMS AS, 2012, DECIS SUPPORT SYST & 72 & 10.3 \\
\hline POYRY E, 2013, ELECTRON COMMER R A & 71 & 11.8 \\
\hline SERAJ M, 2012, J INTERACT MARK & 70 & 10.0 \\
\hline
\end{tabular}




\begin{tabular}{|c|c|c|}
\hline PARENT M, 2011, BUS HORIZONS & 70 & 8.8 \\
\hline CHUA AYK, 2013, J KNOWL MANAG & 67 & 11.2 \\
\hline LIU C, 2014, IEEE T PARALL DISTR & 65 & 13.0 \\
\hline OESTREICHER-SINGER G, 2013, MIS QUART & 64 & 10.7 \\
\hline YANG CW, 2017, INT J DIGIT EARTH & 63 & 31.5 \\
\hline HAJLI MN, 2014, INT J MARKET RES & 60 & 12.0 \\
\hline MCCARTHY J, 2014, INTERNET RES & 59 & 11.8 \\
\hline SHEN J, 2012, J ELECTRON COMMER RE & 56 & 8.0 \\
\hline MARINE-ROIG E, 2015, J DESTIN MARK MANAGE & 54 & 13.5 \\
\hline MURALIDHARAN S, 2011, PUBLIC RELAT REV & 53 & 6.6 \\
\hline KUMAR V, 2015, J MARKETING & 52 & 13.0 \\
\hline MELIAN-GONZALEZ S, 2013, CORNELL HOSP Q & 52 & 8.7 \\
\hline BLAZEVIC V, 2013, J SERV MANAGE & 52 & 8.7 \\
\hline BRAVO-MARQUEZ F, 2014, KNOWL-BASED SYST & 50 & 10.0 \\
\hline LIU SY, 2014, SIGMOD'14: PROCEEDINGS OF THE 2014 ACM SIGMOD IN- & 49 & 9.8 \\
\hline BECHMANN A, 2013, NEW MEDIA SOC & 49 & 8.2 \\
\hline NGAI EWT, 2015, IND MANAGE DATA SYST & 48 & 12.0 \\
\hline BATRINCA B, 2015, AI SOC & 47 & 11.8 \\
\hline WU YC, 2014, IEEE T VIS COMPUT GR & 47 & 9.4 \\
\hline PENTINA I, 2013, COMPUT HUM BEHAV & 47 & 7.8 \\
\hline SPARKS BA, 2016, TOURISM MANAGE & 46 & 15.3 \\
\hline BEREZINA K, 2016, J HOSP MARKET MANAG & 46 & 15.3 \\
\hline JUSSILA JJ, 2014, COMPUT HUM BEHAV & 46 & 9.2 \\
\hline VERHOEF PC, 2013, EUR MANAG J & 46 & 7.7 \\
\hline PALACIOS-MARQUES D, 2015, MANAGE DECIS & 45 & 11.3 \\
\hline GRIFFIS HM, 2014, J MED INTERNET RES & 45 & 9.0 \\
\hline HU YJ, 2015, COMPUT ENVIRON URBAN & 44 & 11.0 \\
\hline KLAUS P, 2013, J SERV MARK & 44 & 7.3 \\
\hline MUNAR AM, 2012, SCAND J HOSP TOUR & 44 & 6.3 \\
\hline ZHANG MM, 2011, ELECTRON MARK & 44 & 5.5 \\
\hline TURCOTTE J, 2015, J COMPUT-MEDIAT COMM & 43 & 10.8 \\
\hline HE W, 2015, INFORM MANAGE-AMSTER & 42 & 10.5 \\
\hline SWANI K, 2014, IND MARKET MANAG & 41 & 8.2 \\
\hline PANIAGUA J, 2014, BUS HORIZONS & 40 & 8.0 \\
\hline OESTREICHER-SINGER G, 2012, MIS QUART & 40 & 5.7 \\
\hline CAIN J, 2011, AM J PHARM EDUC & 40 & 5.0 \\
\hline BRAOJOS-GOMEZ J, 2015, INT J INFORM MANAGE & 39 & 9.8 \\
\hline NGO-YE TL, 2014, DECIS SUPPORT SYST & 39 & 7.8 \\
\hline VERMA R, 2012, CORNELL HOSP Q & 39 & 5.6 \\
\hline GOSSLING S, 2017, J SUSTAIN TOUR & 38 & 19.0 \\
\hline HAJLI N, 2015, TECHNOL FORECAST SOC & 38 & 9.5 \\
\hline EVANS C, 2014, BRIT J EDUC TECHNOL & 38 & 7.6 \\
\hline WHEELER CK, 2011, AESTHET SURG J & 38 & 4.8 \\
\hline WEINBERG BD, 2013, J INTERACT MARK & 37 & 6.2 \\
\hline MILLER AR, 2013, INFORM SYST RES & 37 & 6.2 \\
\hline JANSSEN M, 2014, SOC SCI COMPUT REV & 36 & 7.2 \\
\hline WAMBA SF, 2014, J ORGAN END USER COM & 36 & 7.2 \\
\hline GAL-TZUR A, 2014, TRANSPORT POLICY & 36 & 7.2 \\
\hline KOO C, 2011, INT J INFORM MANAGE & 36 & 4.5 \\
\hline KAPLAN AM, 2016, BUS HORIZONS & 35 & 11.7 \\
\hline GAMBOA AM, 2014, BUS HORIZONS & 35 & 7.0 \\
\hline TIAGO MTPMB, 2014, BUS HORIZONS & 34 & 6.8 \\
\hline COLLINS C, 2013, J PUBLIC TRANSPORT & 34 & 5.7 \\
\hline WANG H, 2016, INFORM SCIENCES & 33 & 11.0 \\
\hline KAUN A, 2014, NEW MEDIA SOC & 33 & 6.6 \\
\hline CURTY RG, 2013, ELECTRON COMMER R A & 33 & 5.5 \\
\hline POWERS T, 2012, J ADVERTISING RES & 33 & 4.7 \\
\hline FELIX R, 2017, J BUS RES & 32 & 16.0 \\
\hline BHARATI P, 2015, J KNOWL MANAG & 32 & 8.0 \\
\hline ZHAO XY, 2015, INT J CONTEMP HOSP M & 32 & 8.0 \\
\hline SUN GD, 2014, IEEE T VIS COMPUT GR & 32 & 6.4 \\
\hline WHELAN G, 2013, J BUS ETHICS & 32 & 5.3 \\
\hline ROBLEK V, 2013, KYBERNETES & 32 & 5.3 \\
\hline
\end{tabular}




\begin{tabular}{|c|c|c|}
\hline HARRIS J, 2012, MIS Q EXEC & 32 & 4.6 \\
\hline GREGOIRE Y, 2015, BUS HORIZONS & 31 & 7.8 \\
\hline POELL T, 2014, INFORM COMMUN SOC & 30 & 6.0 \\
\hline SIGALA M, 2014, CORNELL HOSP Q & 30 & 6.0 \\
\hline WEI YJ, 2011, J ELECTRON COMMER RE & 30 & 3.8 \\
\hline PADILLA-MELENDEZ A, 2013, INT J INFORM MANAGE & 29 & 4.8 \\
\hline MANGOLD WG, 2012, BUS HORIZONS & 29 & 4.1 \\
\hline HEWETT K, 2016, J MARKETING & 28 & 9.3 \\
\hline SCHOLZ J, 2016, BUS HORIZONS & 28 & 9.3 \\
\hline WANG LZ, 2015, IEEE CLOUD COMPUT & 28 & 7.0 \\
\hline ALADWANI AM, 2014, COMPUT HUM BEHAV & 28 & 5.6 \\
\hline YOON S, 2013, BMC PUBLIC HEALTH & 28 & 4.7 \\
\hline OH I, 2012, KOREA OBS & 28 & 4.0 \\
\hline KIRTIS AK, 2011, PROCD SOC BEHV & 28 & 3.5 \\
\hline MORAN E, 2010, J ADVERTISING RES & 28 & 3.1 \\
\hline GUESALAGA R, 2016, IND MARKET MANAG & 27 & 9.0 \\
\hline KOSTKOVA P, 2016, FRONT PUBLIC HEALTH & 27 & 9.0 \\
\hline GRUBER DA, 2015, BUS HORIZONS & 27 & 6.8 \\
\hline LIN CY, 2012, P IEEE & 27 & 3.9 \\
\hline LEE JL, 2014, J MED INTERNET RES & 26 & 5.2 \\
\hline NILSSON E, 2014, J SERV MARK & 26 & 5.2 \\
\hline BRZOZOWSKI MJ, 2009, GROUP 2009 PROCEEDINGS & 26 & 2.6 \\
\hline KILLIAN G, 2015, BUS HORIZONS & 25 & 6.3 \\
\hline REILLY AH, 2014, BUS HORIZONS & 25 & 5.0 \\
\hline KENT ML, 2014, PUBLIC RELAT REV & 25 & 5.0 \\
\hline CARIM L, 2013, PUBLIC RELAT REV & 25 & 4.2 \\
\hline MESKE C, 2013, LECT NOTES BUS INF P & 25 & 4.2 \\
\hline SALDANHA TJV, 2012, J ORG COMP ELECT COM & 25 & 3.6 \\
\hline DONG JQ, 2015, J STRATEGIC INF SYST & 24 & 6.0 \\
\hline HUANG Z, 2015, TECHNOL FORECAST SOC & 24 & 6.0 \\
\hline ZHAO ZZ, 2015, BUS HORIZONS & 24 & 6.0 \\
\hline HARRIGAN P, 2015, AUSTRALAS MARK J & 24 & 6.0 \\
\hline SIGALA M, 2017, CURR ISSUES TOUR & 23 & 11.5 \\
\hline NSOESIE EO, 2014, PREV MED & 23 & 4.6 \\
\hline SARAVANAKUMAR M, 2012, LIFE SCI J & 23 & 3.3 \\
\hline HILL S, 2011, INT J ELECTRON COMM & 23 & 2.9 \\
\hline WHITAKER C, 2017, J MED INTERNET RES & 22 & 11.0 \\
\hline LEE I, 2017, BUS HORIZONS & 22 & 11.0 \\
\hline BUSALIM AH, 2016, INT J INFORM MANAGE & 22 & 7.3 \\
\hline KNOLL J, 2016, INT J ADVERT & 22 & 7.3 \\
\hline AININ S, 2015, IND MANAGE DATA SYST & 22 & 5.5 \\
\hline KOHLI C, 2015, BUS HORIZONS & 22 & 5.5 \\
\hline MCCANN M, 2015, J SMALL BUS ENTERP D & 22 & 5.5 \\
\hline LIBURD JJ, 2013, J HOSP LEIS SPORT TO & 22 & 3.7 \\
\hline USHER WT, 2012, AUST J PRIM HEALTH & 22 & 3.1 \\
\hline SOTO-ACOSTA P, 2017, J TECHNOL TRANSFER & 21 & 10.5 \\
\hline ABBASI A, 2016, J ASSOC INF SYST & 21 & 7.0 \\
\hline RINGELHAN S, 2015, PLOS ONE & 21 & 5.3 \\
\hline ZHU YQ, 2015, BUS HORIZONS & 21 & 5.3 \\
\hline MILES SJ, 2014, BUS HORIZONS & 21 & 4.2 \\
\hline BENSON V, 2014, COMPUT HUM BEHAV & 21 & 4.2 \\
\hline DAHNIL MI, 2014, PROCD SOC BEHV & 21 & 4.2 \\
\hline PERRIGOT R, 2012, J SMALL BUS MANAGE & 21 & 3.0 \\
\hline ASHURST C, 2012, INT J INFORM MANAGE & 21 & 3.0 \\
\hline KAPLAN AM, 2012, BUS HORIZONS-a & 21 & 3.0 \\
\hline SINGARAJU SP, 2016, IND MARKET MANAG & 20 & 6.7 \\
\hline XIE KL, 2016, J TRAVEL TOUR MARK & 20 & 6.7 \\
\hline ZADEH AH, 2014, DECIS SUPPORT SYST & 20 & 4.0 \\
\hline BLAZEVIC V, 2014, J INTERACT MARK & 20 & 4.0 \\
\hline KORSCHUN D, 2013, J BUS RES & 20 & 3.3 \\
\hline
\end{tabular}




\section{Conclusion}

This study has been in the field of analyzing and illustrating the scientific products of the world for 14 Years in the fields of social media and business. The increasing growth of studies began in 2014 . However, in 2018, its steep growth stalled. We are likely to consider this because of the review of subcategories and the exclusion of the word business from the keywords. A large variety of keywords, and of course low density, points to the broadness of the subject. Therefore, it should be dealt with in more depth. This issue can be further explored from two dimensions: Social networking platform and companies by industry. Country collaboration map shows that the United States and China are pioneer in this topic.

\section{References}

Abbasi, A., Sarker, S., \& Chiang, R. H. (2016). Big data research in information systems: Toward an inclusive research agenda. Journal of the Association for Information Systems, 17(2).

Abrahams, A. S., Jiao, J., Wang, G. A., \& Fan, W. (2012). Vehicle defect discovery from social media. Decision Support Systems, 54(1), 87-97.

Ainin, S., Parveen, F., Moghavvemi, S., Jaafar, N. I., \& Mohd Shuib, N. L. (2015). Factors influencing the use of social media by SMEs and its performance outcomes. Industrial Management \& Data Systems, 115(3), 570-588.

Aladwani, A. M. (2014). Gravitating towards Facebook (GoToFB): What it is? and How can it be measured? Computers in Human Behavior, 33, 270-278.

Aral, S., Dellarocas, C., \& Godes, D. (2013). Introduction to the Special Issue - Social Media and Business Transformation: A Framework for Research. Information Systems Research, 24(1), 3-13.

Aria, M., \& Cuccurullo, C. (2017). bibliometrix: An R-tool for comprehensive science mapping analysis. Journal of Informetrics, 11(4), 959-975.

Ashurst, C., Freer, A., Ekdahl, J., \& Gibbons, C. (2012). Exploring IT-enabled innovation: A new paradigm?. International Journal of Information Management, 32(4), 326-336.

Batrinca, B., \& Treleaven, P. C. (2015). Social media analytics: a survey of techniques, tools and platforms. AI \& SOCIETY, 30(1), 89-116.

Bechmann, A., \& Lomborg, S. (2013). Mapping actor roles in social media: Different perspectives on value creation in theories of user participation. New Media \& Society, 15(5), 765-781.

Benson, V., Morgan, S., \& Filippaios, F. (2014). Social career management: Social media and employability skills gap. Computers in Human Behavior, 30, 519-525.

Berezina, K., Bilgihan, A., Cobanoglu, C., \& Okumus, F. (2016). Understanding Satisfied and Dissatisfied Hotel Customers: Text Mining of Online Hotel Reviews. Journal of Hospitality Marketing \& Management, 25(1), 1-24.

Berthon, P. R., Pitt, L. F., Plangger, K., \& Shapiro, D. (2012). Marketing meets Web 2.0, social media, and creative consumers: Implications for international marketing strategy. Business Horizons, 55(3), 261-271.

Bharati, P., Zhang, W., \& Chaudhury, A. (2015). Better knowledge with social media? Exploring the roles of social capital and organizational knowledge management. Journal of Knowledge Management, 19(3), 456-475.

Blazevic, V., Hammedi, W., Garnefeld, I., Rust, R. T., Keiningham, T., Andreassen, T. W., ... Carl, W. (2013). Beyond traditional word-of-mouth. Journal of Service Management, 24(3), 294-313.

Blazevic, V., Wiertz, C., Cotte, J., de Ruyter, K., \& Keeling, D. I. (2014). GOSIP in Cyberspace: Conceptualization and Scale Development for General Online Social Interaction Propensity. Journal of Interactive Marketing, 28(2), 87-100.

Braojos-Gomez, J., Benitez-Amado, J., \& Javier Llorens-Montes, F. (2015). How do small firms learn to develop a social media competence? International Journal of Information Management, 35(4), $443-458$. 
Bravo-Marquez, F., Mendoza, M., \& Poblete, B. (2014). Meta-level sentiment models for big social data analysis. Knowledge-Based Systems, 69, 86-99.

Busalim, A. H., \& Hussin, A. R. C. (2016). Understanding social commerce: A systematic literature review and directions for further research. International Journal of Information Management, 36(6), 1075-1088.

Cain, J., \& Policastri, A. (2011). Using Facebook as an Informal Learning Environment. American Journal of Pharmaceutical Education, 75(10), 207.

Carim, L., \& Warwick, C. (2013). Use of social media for corporate communications by research-funding organisations in the UK. Public Relations Review, 39(5), 521-525.

Chae, B. (Kevin). (2015). Insights from hashtag \#supplychain and Twitter Analytics: Considering Twitter and Twitter data for supply chain practice and research. International Journal of Production Economics, 165, 247-259.

Cheng, M. (2016). Sharing economy: A review and agenda for future research. International Journal of Hospitality Management, 57, 60-70.

Chua, A. Y., \& Banerjee, S. (2013). Customer knowledge management via social media: the case of Starbucks. Journal of Knowledge Management, 17(2), 237-249.

Collins, C., Hasan, S., \& Ukkusuri, S. (2013). A Novel Transit Rider Satisfaction Metric: Rider Sentiments Measured from Online Social Media Data. Journal of Public Transportation, 16(2), 21-45.

Colomo-Palacios, R., Messnarz, R., Siakas, K., Palosi, D., \& Coakley, D. (2014). Using social media as a tool for business improvement and certification of knowledge workers. Journal of Software: Evolution and Process, 26(9), 791-798.

Brzozowski, M. J. (2009, May). WaterCooler: exploring an organization through enterprise social media. In Proceedings of the ACM 2009 international conference on Supporting group work (pp. 219-228). ACM.

Culnan, M. J., McHugh, P. J., \& Zubillaga, J. I. (2010). How large US companies can use Twitter and other social media to gain business value. MIS Quarterly Executive, 9(4).

Dahnil, M. I., Marzuki, K. M., Langgat, J., \& Fabeil, N. F. (2014). Factors Influencing SMEs Adoption of Social Media Marketing. Procedia - Social and Behavioral Sciences, 148, 119-126.

Davenport, T. H., Barth, P. F. P., \& Bean, R. (2012). How 'Big Data' is Different FALL 2012.

Dong, J. Q., \& Wu, W. (2015). Business value of social media technologies: Evidence from online user innovation communities. The Journal of Strategic Information Systems, 24(2), 113-127.

Du, S., \& Vieira, E. T. (2012). Striving for Legitimacy through Corporate Social Responsibility: Insights from Oil Companies. Journal of Business Ethics, 110(4), 413-427.

Evans, C. (2014). Twitter for teaching: Can social media be used to enhance the process of learning? British Journal of Educational Technology, 45(5), 902-915.

Felix, R., Rauschnabel, P. A., \& Hinsch, C. (2017). Elements of strategic social media marketing: A holistic framework. Journal of Business Research, 70, 118-126.

Ferrara, E., De Meo, P., Fiumara, G., \& Baumgartner, R. (2014). Web data extraction, applications and techniques: A survey. Knowledge-Based Systems, 70, 301-323.

Fournier, S., \& Avery, J. (2011). The uninvited brand. Business Horizons, 54(3), 193-207.

Gal-Tzur, A., Grant-Muller, S. M., Kuflik, T., Minkov, E., Nocera, S., \& Shoor, I. (2014). The potential of social media in delivering transport policy goals. Transport Policy, 32, 115-123.

Gamboa, A. M., \& Gonçalves, H. M. (2014). Customer loyalty through social networks: Lessons from Zara on Facebook. Business Horizons, 57(6), 709-717.

Gonçalves Curty, R. (2013). Website features that gave rise to social commerce: a historical analysis. Electronic Commerce Research and Applications, 12(4), 260-279.

Gössling, S. (2017). Tourism, information technologies and sustainability: an exploratory review. Journal of Sustainable Tourism, 25(7), 1024-1041.

Grégoire, Y., Salle, A., \& Tripp, T. M. (2015). Managing social media crises with your customers: The good, the bad, and the ugly. Business Horizons, 58(2), 173-182. 
Griffis, H. M., Kilaru, A. S., Werner, R. M., Asch, D. A., Hershey, J. C., Hill, S., .. Merchant, R. M. (2014). Use of social media across US hospitals: descriptive analysis of adoption and utilization. Journal of Medical Internet Research, 16(11), e264.

Gruber, D. A., Smerek, R. E., Thomas-Hunt, M. C., \& James, E. H. (2015). The real-time power of Twitter: Crisis management and leadership in an age of social media. Business Horizons, 58(2), 163172.

Guesalaga, R. (2016). The use of social media in sales: Individual and organizational antecedents, and the role of customer engagement in social media. Industrial Marketing Management, 54, 71-79.

Hajli, M. N. (2014). A study of the impact of social media on consumers. International Journal of Market Research, 56(3), 387-404.

Hajli, M. N. (2014). The role of social support on relationship quality and social commerce. Technological Forecasting and Social Change, 87, 17-27.

Hajli, N., \& Sims, J. (2015). Social commerce: The transfer of power from sellers to buyers. Technological Forecasting and Social Change, 94, 350-358.

Hanna, R., Rohm, A., \& Crittenden, V. L. (2011). We're all connected: The power of the social media ecosystem. Business Horizons, 54(3), 265-273.

Harrigan, P., Soutar, G., Choudhury, M. M., \& Lowe, M. (2015). Modelling CRM in a social media age. Australasian Marketing Journal (AMJ), 23(1), 27-37.

Harris, J., Ives, B., \& Junglas, I. (2012). IT consumerization: When gadgets turn into enterprise IT tools. MIS Quarterly Executive, 11(3).

Hassan Zadeh, A., \& Sharda, R. (2014). Modeling brand post popularity dynamics in online social networks. Decision Support Systems, 65, 59-68.

He, W., Wu, H., Yan, G., Akula, V., \& Shen, J. (2015). A novel social media competitive analytics framework with sentiment benchmarks. Information \& Management, 52(7), 801-812.

He, W., Zha, S., \& Li, L. (2013). Social media competitive analysis and text mining: A case study in the pizza industry. International Journal of Information Management, 33(3), 464-472.

Hewett, K., Rand, W., Rust, R. T., \& van Heerde, H. J. (2016). Brand Buzz in the Echoverse. Journal of Marketing, 80(3), 1-24.

Hill, S., \& Ready-Campbell, N. (2011). Expert Stock Picker: The Wisdom of (Experts in) Crowds. International Journal of Electronic Commerce, 15(3), 73-102.

Hu, Y., Gao, S., Janowicz, K., Yu, B., Li, W., \& Prasad, S. (2015). Extracting and understanding urban areas of interest using geotagged photos. Computers, Environment and Urban Systems, 54, $240-254$.

Huang, Z., \& Benyoucef, M. (2015). User preferences of social features on social commerce websites: An empirical study. Technological Forecasting and Social Change, 95, 57-72.

Jang, H. J., Sim, J., Lee, Y., \& Kwon, O. (2013). Deep sentiment analysis: Mining the causality between personality-value-attitude for analyzing business ads in social media. Expert Systems with applications, 40(18), 7492-7503.

Janssen, M., \& Zuiderwijk, A. (2014). Infomediary Business Models for Connecting Open Data Providers and Users. Social Science Computer Review, 32(5), 694-711.

Jones, N., Borgman, R., \& Ulusoy, E. (2015). Impact of social media on small businesses. Journal of Small Business and Enterprise Development, 22(4), 611-632.

Jussila, J. J., Kärkkäinen, H., \& Aramo-Immonen, H. (2014). Social media utilization in business-tobusiness relationships of technology industry firms. Computers in Human Behavior, 30, 606-613.

Kaplan, A. M. (2012). If you love something, let it go mobile: Mobile marketing and mobile social media 4x4. Business Horizons, 55(2), 129-139.

Kaplan, A. M., \& Haenlein, M. (2012). The Britney Spears universe: Social media and viral marketing at its best. Business Horizons, 55(1), 27-31.

Kaplan, A. M., \& Haenlein, M. (2016). Higher education and the digital revolution: About MOOCs, SPOCs, social media, and the Cookie Monster. Business Horizons, 59(4), 441-450.

Kaplan, A. M., \& Haenlein, M. (2009). The fairyland of Second Life: Virtual social worlds and how to use them. Business Horizons, 52(6), 563-572. 
Kaplan, A. M., \& Haenlein, M. (2011). Two hearts in three-quarter time: How to waltz the social media/viral marketing dance. Business Horizons, 54(3), 253-263.

Kaplan, A. M., \& Haenlein, M. (2011). The early bird catches the news: Nine things you should know about micro-blogging. Business Horizons, 54(2), 105-113.

Kaplan, A. M., \& Haenlein, M. (2010). Users of the world, unite! The challenges and opportunities of Social Media. Business Horizons, 53(1), 59-68.

Kaun, A., \& Stiernstedt, F. (2014). Facebook time: Technological and institutional affordances for media memories. New Media \& Society, 16(7), 1154-1168.

Kavanaugh, A. L., Fox, E. A., Sheetz, S. D., Yang, S., Li, L. T., Shoemaker, D. J., ... \& Xie, L. (2012). Social media use by government: From the routine to the critical. Government Information Quarterly, 29(4), 480-491.

Kelley School of Business. Accounting and Information Systems Department. (2002). MIS quarterly executive. Accounting and Information Systems Dept., Kelley School of Business, Indiana University.

Kent, M. L., \& Saffer, A. J. (2014). A Delphi study of the future of new technology research in public relations. Public Relations Review, 40(3), 568-576.

Kietzmann, J. H., Hermkens, K., McCarthy, I. P., \& Silvestre, B. S. (2011). Social media? Get serious! Understanding the functional building blocks of social media. Business Horizons, 54(3), 241-251.

Killian, G., \& McManus, K. (2015). A marketing communications approach for the digital era: Managerial guidelines for social media integration. Business Horizons, 58(5), 539-549.

Kim, W., Jeong, O.-R., \& Lee, S.-W. (2010). On social Web sites. Information Systems, 35(2), 215-236.

Kirtiş, A. K., \& Karahan, F. (2011). To Be or Not to Be in Social Media Arena as the Most Cost-Efficient Marketing Strategy after the Global Recession. Procedia - Social and Behavioral Sciences, 24, 260268.

Klaus, P. (2013). The case of Amazon.com: towards a conceptual framework of online customer service experience (OCSE) using the emerging consensus technique (ECT). Journal of Services Marketing, 27(6), 443-457.

Knoll, J. (2016). Advertising in social media: a review of empirical evidence. International Journal of Advertising, 35(2), 266-300.

Kohli, C., Suri, R., \& Kapoor, A. (2015). Will social media kill branding? Business Horizons, 58(1), 3544.

Koo, C., Wati, Y., \& Jung, J. J. (2011). Examination of how social aspects moderate the relationship between task characteristics and usage of social communication technologies (SCTs) in organizations. International Journal of Information Management, 31(5), 445-459.

Korschun, D., \& Du, S. (2013). How virtual corporate social responsibility dialogs generate value: A framework and propositions. Journal of Business Research, 66(9), 1494-1504.

Kostkova, P., Brewer, H., de Lusignan, S., Fottrell, E., Goldacre, B., Hart, G., ... Tooke, J. (2016). Who Owns the Data? Open Data for Healthcare. Frontiers in Public Health, 4, 7.

Kumar, V. (2015). Evolution of Marketing as a Discipline: What Has Happened and What to Look Out For. Journal of Marketing, 79(1), 1-9.

Kwok, L., \& Yu, B. (2013). Spreading Social Media Messages on Facebook. Cornell Hospitality Quarterly, 54(1), 84-94.

Lariscy, R. W., Avery, E. J., Sweetser, K. D., \& Howes, P. (2009). An examination of the role of online social media in journalists' source mix. Public Relations Review, 35(3), 314-316.

Lee, I. (2017). Big data: Dimensions, evolution, impacts, and challenges. Business Horizons, 60(3), 293303.

Lee, J. L., DeCamp, M., Dredze, M., Chisolm, M. S., \& Berger, Z. D. (2014). What are health-related users tweeting? A qualitative content analysis of health-related users and their messages on twitter. Journal of Medical Internet Research, 16(10), e237.

Li, L., Goodchild, M. F., \& Xu, B. (2013). Spatial, temporal, and socioeconomic patterns in the use of Twitter and Flickr. Cartography and Geographic Information Science, 40(2), 61-77. 
Liang, T.-P., \& Turban, E. (2011). Introduction to the Special Issue Social Commerce: A Research Framework for Social Commerce. International Journal of Electronic Commerce, 16(2), 5-14.

Liburd, J. J., \& Christensen, I.-M. F. (2013). Using web 2.0 in higher tourism education. Journal of Hospitality, Leisure, Sport \& Tourism Education, 12(1), 99-108.

Lin, C.-Y., Wu, L., Wen, Z., Tong, H., Griffiths-Fisher, V., Shi, L., \& Lubensky, D. (2012). Social Network Analysis in Enterprise. Proceedings of the IEEE, 100(9), 2759-2776.

Lin, K.-Y., \& Lu, H.-P. (2011). Intention to Continue Using Facebook Fan Pages from the Perspective of Social Capital Theory. Cyberpsychology, Behavior, and Social Networking, 14(10), 565-570.

Liu, C., Chen, J., Yang, L. T., Zhang, X., Yang, C., Ranjan, R., \& Rao, K. (2014). Authorized Public Auditing of Dynamic Big Data Storage on Cloud with Efficient Verifiable Fine-Grained Updates. IEEE Transactions on Parallel and Distributed Systems, 25(9), 2234-2244.

Liu, S., Wang, S., Zhu, F., Zhang, J., \& Krishnan, R. (2014). HYDRA. In Proceedings of the 2014 ACM SIGMOD international conference on Management of data - SIGMOD '14 (pp. 51-62). New York, New York, USA: ACM Press.

Lizhe Wang, \& Ranjan, R. (2015). Processing Distributed Internet of Things Data in Clouds. IEEE Cloud Computing, 2(1), 76-80.

Lu, Y., Wang, F., \& Maciejewski, R. (2014). Business intelligence from social media: A study from the vast box office challenge. IEEE computer graphics and applications, 34(5), 58-69.

Luo, X., Zhang, J., \& Duan, W. (2013). Social Media and Firm Equity Value. Information Systems Research, 24(1), 146-163.

Malthouse, E. C., Haenlein, M., Skiera, B., Wege, E., \& Zhang, M. (2013). Managing Customer Relationships in the Social Media Era: Introducing the Social CRM House. Journal of Interactive Marketing, 27(4), 270-280.

Mangold, W. G., \& Faulds, D. J. (2009). Social media: The new hybrid element of the promotion mix. Business Horizons, 52(4), 357-365.

Mangold, W. G., \& Smith, K. T. (2012). Selling to Millennials with online reviews. Business Horizons, 55(2), 141-153.

Marine-Roig, E., \& Anton Clavé, S. (2015). Tourism analytics with massive user-generated content: A case study of Barcelona. Journal of Destination Marketing \& Management, 4(3), 162-172.

McCann, M., \& Barlow, A. (2015). Use and measurement of social media for SMEs. Journal of Small Business and Enterprise Development, 22(2), 273-287.

McCarthy, J., Rowley, J., Jane Ashworth, C., \& Pioch, E. (2014). Managing brand presence through social media: the case of UK football clubs. Internet Research, 24(2), 181-204.

Melián-González, S., Bulchand-Gidumal, J., \& González López-Valcárcel, B. (2013). Online Customer Reviews of Hotels. Cornell Hospitality Quarterly, 54(3), 274-283.

Meske, C., \& Stieglitz, S. (2013). Adoption and Use of Social Media in Small and Medium-Sized Enterprises (pp. 61-75).

Michaelidou, N., \& Siamagka, N. T. (2011). Usage, barriers and measurement of social media marketing: An exploratory investigation of small and medium B2B brands. Industrial Marketing Management, 40(7), 1153-1159.

Miles, S. J., \& Mangold, W. G. (2014). Employee voice: Untapped resource or social media time bomb? Business Horizons, 57(3), 401-411.

Miller, A. R., \& Tucker, C. (2013). Active Social Media Management: The Case of Health Care. Information Systems Research, 24(1), 52-70.

Moran, E., \& Gossieaux, F. (2010). Marketing in a Hyper-Social World. Journal of Advertising Research, $50(3), 232-239$.

Munar, A. M. (2012). Social Media Strategies and Destination Management. Scandinavian Journal of Hospitality and Tourism, 12(2), 101-120.

Munar, A. M., \& Jacobsen, J. K. S. (2014). Motivations for sharing tourism experiences through social media. Tourism Management, 43, 46-54. 
Muralidharan, S., Dillistone, K., \& Shin, J.-H. (2011). The Gulf Coast oil spill: Extending the theory of image restoration discourse to the realm of social media and beyond petroleum. Public Relations Review, 37(3), 226-232.

Ngai, E. W. T., Moon, K. K., Lam, S. S., Chin, E. S. K., \& Tao, S. S. C. (2015). Social media models, technologies, and applications. Industrial Management \& Data Systems, 115(5), 769-802.

Ngo-Ye, T. L., \& Sinha, A. P. (2014). The influence of reviewer engagement characteristics on online review helpfulness: A text regression model. Decision Support Systems, 61, 47-58.

Nilsson, E., \& Ballantyne, D. (2014). Reexamining the place of servicescape in marketing: a servicedominant logic perspective. Journal of Services Marketing, 28(5), 374-379.

Nsoesie, E. O., Kluberg, S. A., \& Brownstein, J. S. (2014). Online reports of foodborne illness capture foods implicated in official foodborne outbreak reports. Preventive Medicine, 67, 264-269.

Oestreicher-Singer, G., \& Zalmanson, L. (2013). Content or community? A digital business strategy for content providers in the social age. MIS quarterly, 591-616.

Oestreicher-Singer, G., \& Sundararajan, A. (2012). Recommendation Networks and the Long Tail of Electronic Commerce. MIS Quarterly, 36(1), 65.

Oh, I., \& Park, G. S. (2012). From B2C to B2B: Selling Korean pop music in the age of new social media. Korea Observer, 43(3), 365-397.

Padilla-Meléndez, A., \& del Águila-Obra, A. R. (2013). Web and social media usage by museums: Online value creation. International Journal of Information Management, 33(5), 892-898.

Palacios-Marqués, D., Merigó, J. M., \& Soto-Acosta, P. (2015). Online social networks as an enabler of innovation in organizations. Management Decision, 53(9), 1906-1920.

Paniagua, J., \& Sapena, J. (2014). Business performance and social media: Love or hate? Business Horizons, 57(6), 719-728.

Pantelidis, I. S. (2010). Electronic Meal Experience: A Content Analysis of Online Restaurant Comments. Cornell Hospitality Quarterly, 51(4), 483-491.

Parent, M., Plangger, K., \& Bal, A. (2011). The new WTP: Willingness to participate. Business Horizons, 54(3), 219-229.

Pentina, I., Zhang, L., \& Basmanova, O. (2013). Antecedents and consequences of trust in a social media brand: A cross-cultural study of Twitter. Computers in Human Behavior, 29(4), 1546-1555.

Perrigot, R., Kacker, M., Basset, G., \& Cliquet, G. (2012). Antecedents of Early Adoption and Use of Social Media Networks for Stakeholder Communications: Evidence from Franchising*. Journal of Small Business Management, 50(4), 539-565.

Piotrowicz, W., \& Cuthbertson, R. (2014). Introduction to the Special Issue Information Technology in Retail: Toward Omnichannel Retailing. International Journal of Electronic Commerce, 18(4), 5-16.

Poell, T. (2014). Social media and the transformation of activist communication: exploring the social media ecology of the 2010 Toronto G20 protests. Information, Communication \& Society, 17(6), 716731.

Powers, T., Advincula, D., Austin, M. S., Graiko, S., \& Snyder, J. (2012). Digital and Social Media In the Purchase Decision Process. Journal of Advertising Research, 52(4), 479-489.

Pöyry, E., Parvinen, P., \& Malmivaara, T. (2013). Can we get from liking to buying? Behavioral differences in hedonic and utilitarian Facebook usage. Electronic Commerce Research and Applications, 12(4), 224-235.

Rapp, A., Beitelspacher, L. S., Grewal, D., \& Hughes, D. E. (2013). Understanding social media effects across seller, retailer, and consumer interactions. Journal of the Academy of Marketing Science, 41(5), $547-566$.

Ravi, K., \& Ravi, V. (2015). A survey on opinion mining and sentiment analysis: Tasks, approaches and applications. Knowledge-Based Systems, 89, 14-46.

Reilly, A. H., \& Hynan, K. A. (2014). Corporate communication, sustainability, and social media: It's not easy (really) being green. Business Horizons, 57(6), 747-758.

Shen, J. (2012). Social comparison, social presence, and enjoyment in the acceptance of social shopping websites. Journal of Electronic Commerce Research, 13(3), 198. 
Ringelhan, S., Wollersheim, J., \& Welpe, I. M. (2015). I Like, I Cite? Do Facebook Likes Predict the Impact of Scientific Work? PLOS ONE, 10(8), e0134389.

Rishika, R., Kumar, A., Janakiraman, R., \& Bezawada, R. (2013). The Effect of Customers' Social Media Participation on Customer Visit Frequency and Profitability: An Empirical Investigation. Information Systems Research, 24(1), 108-127.

Roblek, V., Pejić Bach, M., Meško, M., \& Bertoncelj, A. (2013). The impact of social media to value added in knowledge-based industries. Kybernetes, 42(4), 554-568.

Saldanha, T. J. V., \& Krishnan, M. S. (2012). Organizational Adoption of Web 2.0 Technologies: An Empirical Analysis. Journal of Organizational Computing and Electronic Commerce, 22(4), 301333.

Sashi, C. M. (2012). Customer engagement, buyer-seller relationships, and social media. Management Decision, 50(2), 253-272.

Saxton, G. D., Oh, O., \& Kishore, R. (2013). Rules of Crowdsourcing: Models, Issues, and Systems of Control. Information Systems Management, 30(1), 2-20.

Scholz, J., \& Smith, A. N. (2016). Augmented reality: Designing immersive experiences that maximize consumer engagement. Business Horizons, 59(2), 149-161.

Scott, S. V., \& Orlikowski, W. J. (2012). Reconuring relations of accountability: Materialization of social media in the travel sector. Accounting, Organizations and Society, 37(1), 26-40.

Seraj, M. (2012). We Create, We Connect, We Respect, Therefore We Are: Intellectual, Social, and Cultural Value in Online Communities. Journal of Interactive Marketing, 26(4), 209-222.

Sigala, M. (2017). Collaborative commerce in tourism: implications for research and industry. Current Issues in Tourism, 20(4), 346-355.

Sigala, M. (2014). Customer Involvement in Sustainable Supply Chain Management. Cornell Hospitality Quarterly, 55(1), 76-88.

Singaraju, S. P., Nguyen, Q. A., Niininen, O., \& Sullivan-Mort, G. (2016). Social media and value cocreation in multi-stakeholder systems: A resource integration approach. Industrial Marketing Management, 54, 44-55.

Sotiriadis, M. D., \& van Zyl, C. (2013). Electronic word-of-mouth and online reviews in tourism services: the use of twitter by tourists. Electronic Commerce Research, 13(1), 103-124.

Soto-Acosta, P., Popa, S., \& Palacios-Marqués, D. (2017). Social web knowledge sharing and innovation performance in knowledge-intensive manufacturing SMEs. The Journal of Technology Transfer, 42(2), 425-440.

Sparks, B. A., So, K. K. F., \& Bradley, G. L. (2016). Responding to negative online reviews: The effects of hotel responses on customer inferences of trust and concern. Tourism Management, 53, 74-85.

Sun, G., Wu, Y., Liu, S., Peng, T.-Q., Zhu, J. J. H., \& Liang, R. (2014). EvoRiver: Visual Analysis of Topic Coopetition on Social Media. IEEE Transactions on Visualization and Computer Graphics, 20(12), 1753-1762.

Swani, K., Brown, B. P., \& Milne, G. R. (2014). Should tweets differ for B2B and B2C? An analysis of Fortune 500 companies' Twitter communications. Industrial Marketing Management, 43(5), 873881.

Tiago, M. T. P. M. B., \& Veríssimo, J. M. C. (2014). Digital marketing and social media: Why bother? Business Horizons, 57(6), 703-708.

Trainor, K. J., Andzulis, J. (Mick), Rapp, A., \& Agnihotri, R. (2014). Social media technology usage and customer relationship performance: A capabilities-based examination of social CRM. Journal of Business Research, 67(6), 1201-1208.

Turcotte, J., York, C., Irving, J., Scholl, R. M., \& Pingree, R. J. (2015). News Recommendations from Social Media Opinion Leaders: Effects on Media Trust and Information Seeking. Journal of Computer-Mediated Communication, 20(5), 520-535.

Usher, W. T. (2012). Australian health professionals' social media (Web 2.0) adoption trends: early 21st century health care delivery and practice promotion. Australian Journal of Primary Health, 18(1), 31. 
Verhoef, P. C., Kannan, P. K., \& Inman, J. J. (2015). From Multi-Channel Retailing to Omni-Channel Retailing: Introduction to the Special Issue on Multi-Channel Retailing. Journal of Retailing, 91(2), 174-181.

Verhoef, P. C., \& Lemon, K. N. (2013). Successful customer value management: Key lessons and emerging trends. European Management Journal, 31(1), 1-15.

Verma, R., Stock, D., \& McCarthy, L. (2012). Customer Preferences for Online, Social Media, and Mobile Innovations in the Hospitality Industry. Cornell Hospitality Quarterly, 53(3), 183-186.

Wamba, S. F., \& Carter, L. (2016). Social media tools adoption and use by SMEs: An empirical study. In Social media and Networking: Concepts, methodologies, tools, and applications (pp. 791-806). IGI Global.

Wang, H., Xu, Z., Fujita, H., \& Liu, S. (2016). Towards felicitous decision making: An overview on challenges and trends of Big Data. Information Sciences, 367-368, 747-765.

Wei, Y., Straub, D. W., \& Poddar, A. (2011). The power of many: an assessment of managing internet group purchasing. Journal of Electronic Commerce Research, 12(1), 19.

Weinberg, B. D., de Ruyter, K., Dellarocas, C., Buck, M., \& Keeling, D. I. (2013). Destination Social Business: Exploring an Organization's Journey with Social Media, Collaborative Community and Expressive Individuality. Journal of Interactive Marketing, 27(4), 299-310.

Weinberg, B. D., \& Pehlivan, E. (2011). Social spending: Managing the social media mix. Business Horizons, 54(3), 275-282.

Wheeler, C. K., Said, H., Prucz, R., Rodrich, R. J., \& Mathes, D. W. (2011). Social Media in Plastic Surgery Practices: Emerging Trends in North America. Aesthetic Surgery Journal, 31(4), 435-441.

Whelan, G., Moon, J., \& Grant, B. (2013). Corporations and Citizenship Arenas in the Age of Social Media. Journal of Business Ethics, 118(4), 777-790.

Whitaker, C., Stevelink, S., \& Fear, N. (2017). The Use of Facebook in Recruiting Participants for Health Research Purposes: A Systematic Review. Journal of Medical Internet Research, 19(8), e290.

Wu, Y., Liu, S., Yan, K., Liu, M., \& Wu, F. (2014). OpinionFlow: Visual Analysis of Opinion Diffusion on Social Media. IEEE Transactions on Visualization and Computer Graphics, 20(12), 1763-1772.

Xiang, Z., Magnini, V. P., \& Fesenmaier, D. R. (2015). Information technology and consumer behavior in travel and tourism: Insights from travel planning using the internet. Journal of Retailing and Consumer Services, 22, 244-249.

Xie, K. L., Chen, C., \& Wu, S. (2016). Online Consumer Review Factors Affecting Offline Hotel Popularity: Evidence from Tripadvisor. Journal of Travel \& Tourism Marketing, 33(2), 211-223.

Xie, K. L., Zhang, Z., \& Zhang, Z. (2014). The business value of online consumer reviews and management response to hotel performance. International Journal of Hospitality Management, 43, 1-12.

Yang, C., Huang, Q., Li, Z., Liu, K., \& Hu, F. (2017). Big Data and cloud computing: innovation opportunities and challenges. International Journal of Digital Earth, 10(1), 13-53.

Yoon, S., \& Lam, T.-H. (2013). The illusion of righteousness: corporate social responsibility practices of the alcohol industry. BMC Public Health, 13(1), 630.

Yu, Y., \& Cao, Q. (2013). The impact of social and conventional media on firm equity value: A sentiment analysis approach. Decision Support Systems, 55(4), 919-926.

Zhang, M., Jansen, B. J., \& Chowdhury, A. (2011). Business engagement on Twitter: a path analysis. Electronic Markets, 21(3), 161-175.

Zhao, X. (Roy), Wang, L., Guo, X., \& Law, R. (2015). The influence of online reviews to online hotel booking intentions. International Journal of Contemporary Hospitality Management, 27(6), 13431364.

Zhao, Z., \& Balagué, C. (2015). Designing branded mobile apps: Fundamentals and recommendations. Business Horizons, 58(3), 305-315.

Zhou, L., Zhang, P., \& Zimmermann, H. D. (2013). Social commerce research: An integrated view. Electronic commerce research and applications, 12(2), 61-68.

Zhu, Y.-Q., \& Chen, H.-G. (2015). Social media and human need satisfaction: Implications for social media marketing. Business Horizons, 58(3), 335-345. 
(C) 2019 by the authors; licensee Growing Science, Canada. This is an open access article distributed under the terms and conditions of the Creative Commons Attribution (CC-BY) license (http://creativecommons.org/licenses/by/4.0/). 